\title{
On Design of CIC Decimators
}

\author{
Gordana Jovanovic Dolecek and Javier Diaz-Carmona \\ Institute INAOE Puebla, Institute ITC Celaya \\ Mexico
}

\section{Introduction}

The process of changing sampling rate of a signal is called sampling rate conversion (SRC). Systems that employ multiple sampling rates in the processing of digital signals are called multirate digital signal processing systems.

Multirate systems have different applications, such as efficient filtering, subband coding, audio and video signals, analog/digital conversion, software defined radio and communications, among others (Jovanovic Dolecek, 2002).

The reduction of a sampling rate is called decimation and consists of two stages: filtering and downsampling. If signal is not properly bandlimited the overlapping of the repeated replicas of the original spectrum occurs. This effect is called aliasing and may destroy the useful information of the decimated signal. That is why we need filtering to avoid this unwanted effect.

The most simple decimation filter is comb filter which does not require multipliers. One efficient implementation of this filter is called CIC (Cascaded-Integrator-Comb) filter proposed by Hogenauer (Hogenauer, 1981). Because of the popularity of this structure many authors also call the comb filter as CIC filter. In this chapter we will use term CIC filter. Due to its simplicity, the CIC filter is usually used in the first stage of decimation. However, the filter exhibits a high passband droop and a low attenuation in so called folding bands (bands around the zeros of CIC filter), which can be not acceptable in different applications. During last several years the improvement of the CIC filter characteristics attracted many researchers. Different methods have been proposed to improve the characteristics of the CIC filters, keeping its simplicity.

In this chapter we present different proposed methods to improve CIC magnitude characteristics illustrated with examples and MATLAB programs.

The rest of the chapter is organized in the following way. Next Section describes the CIC filter. Section 3 introduces the methods for the CIC passband improvement followed by the Section 4 which presents the methods for the CIC stopband improvement. The methods for both, the CIC passband and stopband improvements are described in Section 5.

\section{CIC filter}

CIC (Cascaded-Integrator-Comb) filter (Hogenauer, 1981) is widely used as the decimation filter due to its simplicity; it requires no multiplication or coefficient storage but rather only additions/subtractions. This filter consists of two main sections, cascaded integrators and combs, separated by a down-sampler, as shown in Fig. 1. 


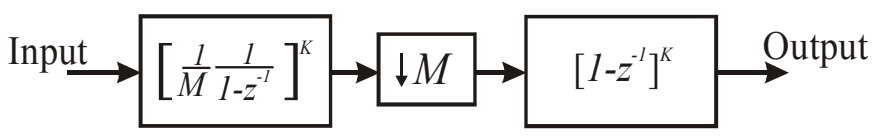

Fig. 1. CIC filter.

The transfer function of the resulting decimation filter, also known as a RRS (recursive running sum) or comb filter is given by

$$
H_{\text {comb }}(z)=\left[\frac{1}{M}\left(\frac{1-z^{-M}}{1-z^{-1}}\right)\right]^{K},
$$

where $M$ is the decimation factor, and $K$ is the number of the stages. The transfer function in (1) will be also referred to as the comb filter. The integrator section works at the higher input data rate thereby resulting in higher chip area and higher power dissipation for this section. In order to resolve this problem the non-recursive structure of Eq. (1) can be used (Aboushady et al., 2001), (Gao at al., 2000),

$$
H(z)=\left[\frac{1}{M}\right]^{K}\left[1+z^{-1}+z^{-2}+\ldots+z^{-(M-1)}\right]^{K} .
$$

Implementing $H(z)$ of Eq. (2) in a polyphase form, the filtering at the high input rate can be moved to the lower rate. In this chapter we do not discuss the CIC implementation issues.

\subsection{Magnitude characteristic}

The magnitude characteristic of the comb decimator must satisfy two requirements:

- To have a low droop in the frequency band defined by the passband frequency $\omega_{p}$ in order to preserve the signal after decimation.

- To have a high attenuations in so called folding bands, i. e. the bands around of the zeros of the comb filter,

$$
\left[\frac{2 \pi i}{M}-\omega_{p} ; \frac{2 \pi i}{M}+\omega_{p}\right], \text { for } i=\left\{\begin{array}{cl}
1, \ldots, M / 2 & \text { for } \quad M \text { even } \\
1, \ldots,(M-1) / 2 & \text { for } \quad M \text { odd }
\end{array}\right.
$$

We define the passband frequency as the frequency where the worst case of passband droop occurs, (Kwentus, Willson, 1997),

$$
\omega_{p}=\frac{\pi}{M R},
$$

where $R$ is the decimation stage that follows the CIC decimation stage, and that is usually much less than $M$.

The magnitude response of the comb filter exhibits a linear-phase, lowpass characteristic which can be expressed as

$$
\left|H_{\text {comb }}\left(e^{j \omega}\right)\right|=\left|\frac{1}{M} \frac{\sin (\omega M / 2)}{\sin (\omega / 2)}\right|^{K} .
$$


Figure 2.a shows the magnitude characteristics in $\mathrm{dB}$ for $M=8$ and the values of $K=1,3$, and 5 .

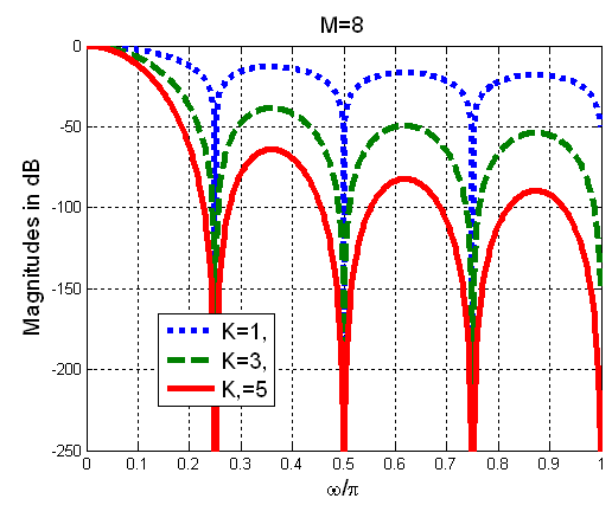

a. Overall magnitude responses.

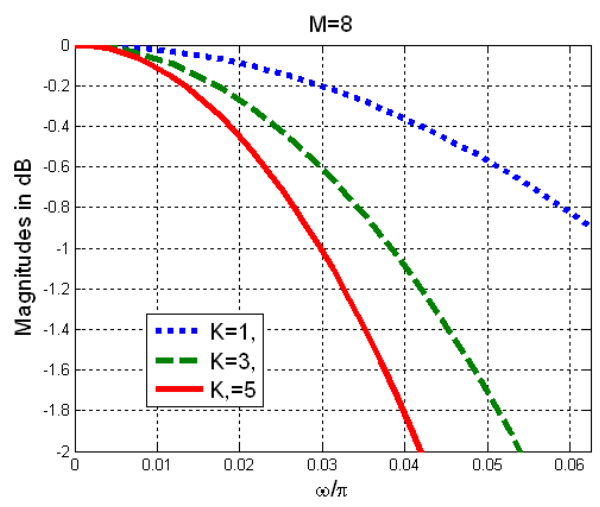

b. Passband zooms.

Fig. 2. Magnitude responses of comb filters.

Note that the attenuations in the folding bands are increased by increasing the numbers of stages. However, an increasing in the number of stages results in the increasing of the passband droop as shown in Fig. 2.b. In the continuation we will consider different methods to improve the comb magnitude characteristics keeping its simplicity.

\section{Methods for the passband improvement}

The motivation behind the compensation methods is to appropriately modify the original CIC characteristic in the desired passband such that the compensator filter has as low complexity as possible. Different methods have been proposed to compensate for the CIC passband droop. We classify the methods as the methods for the narrowband compensation $(R>2)$, and the methods for the wideband compensation $(R=2)$. Methods specified in (Fernandez-Vazquez \& Jovanovic Dolecek, 2009, 2011), (Kim et al. 2006) employ optimization techniques, whereby the resulting compensation filters require multipliers. The method described in (Yeung \& Chan, 2004) suggests the multiplierless design of a second order compensation filter where the filter coefficients are expressed as a sum of power of two (SOPOT) and are computed using the random search algorithm. The simple multiplierless compensator with only one parameter, which depends on the number of the stages $K$ of the CIC filter, is proposed in (Jovanovic Dolecek \& Mitra, 2008). This filter provides a good compensation in a narrow passband. The wide-band compensators have been recently proposed in (Jovanovic Dolecek, 2009), and (Jovanovic Dolecek \& Dolecek, 2010).

We define the following desirable CIC compensator properties:

- The proposed filter should work at a low sampling rate;

- Multiplierless design and a second order at low rate;

- Simple design i.e., that it is not necessary redesign the filter for new values of $M$ and $K$;

- That the compensation filter practically does not depends on the decimation factor $M$. This is a very desirable characteristic because the compensator remains the same across different values of $M$, provided that the value of $K$ stays the same. 


\subsection{Narrowband CIC compensation}

We describe here the compensation filter (Jovanovic Dolecek \& Mitra, 2008) because this filter satisfies all the properties mentioned previously.

Consider a filter with the magnitude response

$$
\left|G\left(e^{j \omega}\right)\right|=\left|1+2^{-b} \sin ^{2}(\omega M / 2)\right|,
$$

where $b$ is a integer parameter.

Using the well known relation

$$
\sin ^{2}(\alpha)=(1-\cos (2 \alpha)) / 2,
$$

the corresponding transfer function can be expressed as

$$
G\left(z^{M}\right)=-2^{-(b+2)}\left[1-\left(2^{b+2}+2\right) z^{-M}+z^{-2 M}\right] .
$$

Denoting

$$
A=-2^{-(b+2)} ; B=-\left(2^{b+2}+2\right),
$$

we arrive at

$$
G\left(z^{M}\right)=A\left[1-B z^{-M}+z^{-2 M}\right]
$$

The compensator filter has the scaling factor $A$ and a single coefficient $B$ which requires only one adder. Additionally, the compensator can be implemented at a lower rate after the downsampling by $M$ by making use of the multirate identity (Jovanovic Dolecek, 2002), becoming a second order filter,

$$
G(z)=A\left[1-B z^{-1}+z^{-2}\right] .
$$

In that way the filter does not depend on the decimation factor $M$ but only on the number of the stages $K$ which defines the parameter $b$ in (9). Table 1 shows typical values for $b$ at different values of $K$.

\begin{tabular}{|c|c|}
\hline Parameter $\boldsymbol{K}$ & Parameter $\boldsymbol{b}, \boldsymbol{R}=\mathbf{8}$ \\
\hline 2 & 2 \\
\hline 3 & 2 \\
\hline 4 & 1 \\
\hline 5 & 0 \\
\hline 6 & 0 \\
\hline
\end{tabular}

Table 1. Typical parameters $b$ for different values of $K$.

The overall transfer function of the cascaded CIC and compensator is

$$
H(z)=H_{\text {comb }}(z) G\left(z^{M}\right),
$$

where $H_{\text {comb }}(z)$ and $G\left(z^{M}\right)$ are given in (1) and (10), respectively. 
Example 1: We compensate the CIC filter with $M=16$ and $K=5$. From Table 1 we have $b=0$. The passband characteristics of the compensator, along with that of the compensated CIC and the CIC filters, are shown in Fig.3.

\subsection{Wideband $\mathrm{CIC}$ compensation}

We turn now our attention to the wideband compensators satisfying the desirable characteristics previously mentioned.

In (Jovanovic Dolecek, 2009) a novel decimation filter

$$
G\left(z^{M}\right)=G_{c}^{K_{1}}\left(z^{M}\right),
$$

is proposed, where $K_{1}$ is the parameter that depends on the number of cascaded CIC filters K,

$$
K_{1}=\left\{\begin{array}{ccc}
K & \text { for } & 1<K \leq 3 \\
K-1 & \text { for } & K>3
\end{array},\right.
$$

and

$$
G_{c}\left(z^{M}\right)=-2^{-4}\left[z^{-M}-\left(2^{4}+2\right) z^{-2 M}+z^{-3 M}\right] .
$$

The coefficients of the filter (15) are obtained using the condition that the compensator magnitude characteristic has the value 1 for $\omega=0$ and minimizing the squared error in the passband. Finally, the coefficients thus obtained are rounded using the rounding constant $r=2^{-6}$.

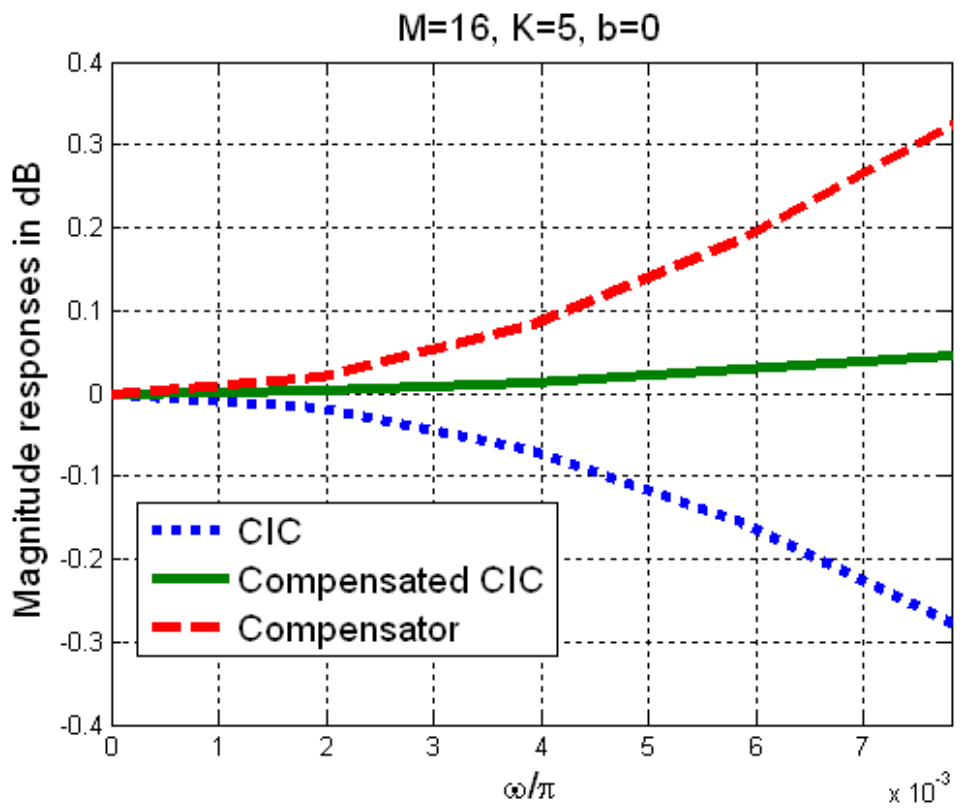

Fig. 3. Magnitude responses of CIC, Compensator and cascaded CIC-compensator. 
The total number of additions depends on $K$, as given by

$$
N_{\text {add }}=\left\{\begin{array}{ccc}
3 K & \text { for } & K \leq 3 \\
3 K-3 & \text { for } & K>3
\end{array}\right. \text {. }
$$

This filter can be moved to a lower rate becoming

$$
G(z)=-2^{-4}\left[z^{-1}-\left(2^{4}+2\right) z^{-2}+z^{-3}\right] .
$$

The overall transfer function of the compensated CIC filter, obtained from (1) and (13)-(15) is as follows

$$
H(z)=H_{\text {comb }}(z) G\left(z^{M}\right)=H_{\text {comb }}(z) G_{c}^{K_{1}}\left(z^{M}\right) .
$$

Note that the filter (17) does not depend on the decimation factor $M$. Additionally, the filter (17) has a very interesting property i.e. it does not depend on $K$ and its structure remains the same for all values of $K$ and $M$. However, the number of the cascaded compensators $K_{1}$ depends on the parameter $K$, as indicated in (14). The method is illustrated in the following example.

Example 2: In this example we compensate the CIC filter with $M=20$ and $K=5$. From (14) it follows that $K_{1}=4$. The magnitude responses of the compensated CIC, along with the responses of the compensator and CIC filters, are shown in Fig.4. From (16) the total number of adders in compensator $3 K-3$, equal 12.

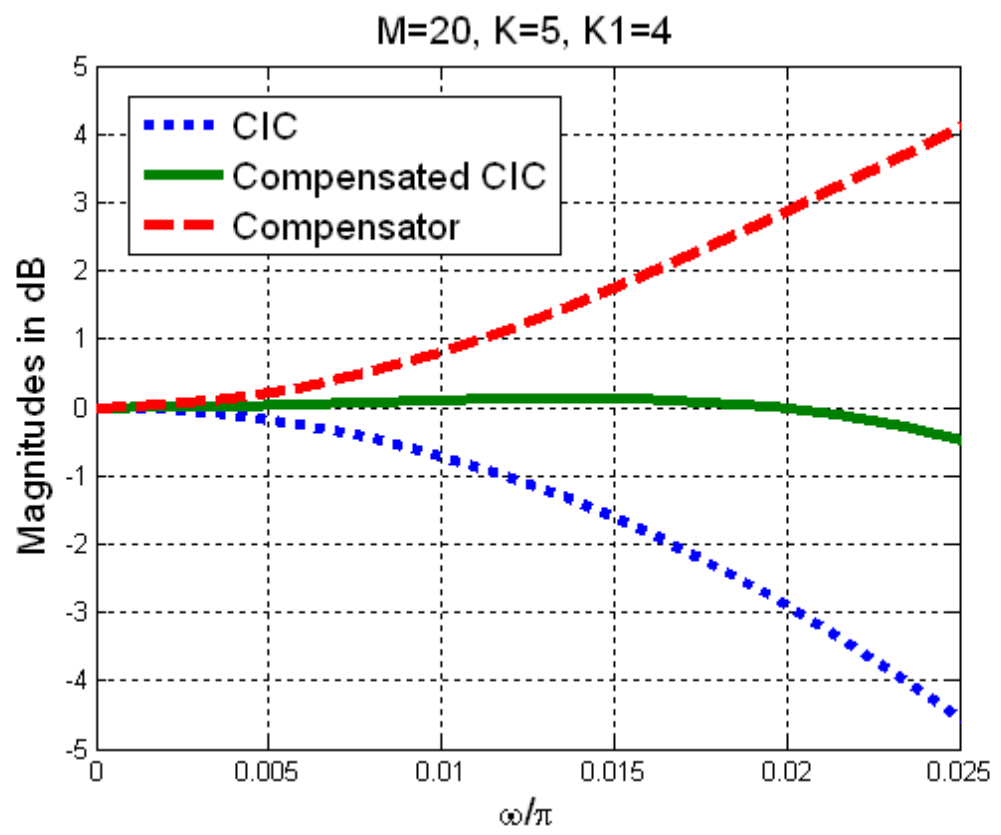

Fig. 4. Wideband compensation method (Jovanovic Dolecek, 2009). 
Example 3: In this example we apply the compensator from (Jovanovic Dolecek, 2009) to the CIC filter with $M=25$ and $K=2$; in this case $K_{1}=2$. The required number of adders for the decimator is $3 K=6$. Figure 5 shows the corresponding magnitude responses.

We will refer here the method from (Jovanovic Dolecek, 2009) as the Compensation method 1. Another simple wideband multiplierless compensator has been proposed in (Jovanovic Dolecek \& Dolecek, 2010). The goal put in it, was that the resulting passband deviation be less than $0.4 \mathrm{~dB}$, and to decrease the number of adders comparing with the Compensation method 1.

To this end the following filter has been proposed,

$$
H_{c}\left(z^{M}\right)=b z^{-M}+a z^{-2 M}+b z^{-3 M},
$$

with the corresponding magnitude response

$$
\left|H_{c}(\omega)\right|=|2 b \cos (M \omega)+a| .
$$

The coefficients $a$ and $b$, obtained in (Jovanovic Dolecek \& Dolecek, 2010), are as follows

$$
\begin{aligned}
& b=0.5\left[1-\frac{\alpha M^{K} \sin ^{K}(\pi / 4 M)}{\sin ^{K}(\pi / 4)}\right] . \\
& a=1-\left[1-\frac{\alpha M^{K} \sin ^{K}(\pi / 4 M)}{\sin ^{K}(\pi / 4)}\right] .
\end{aligned}
$$

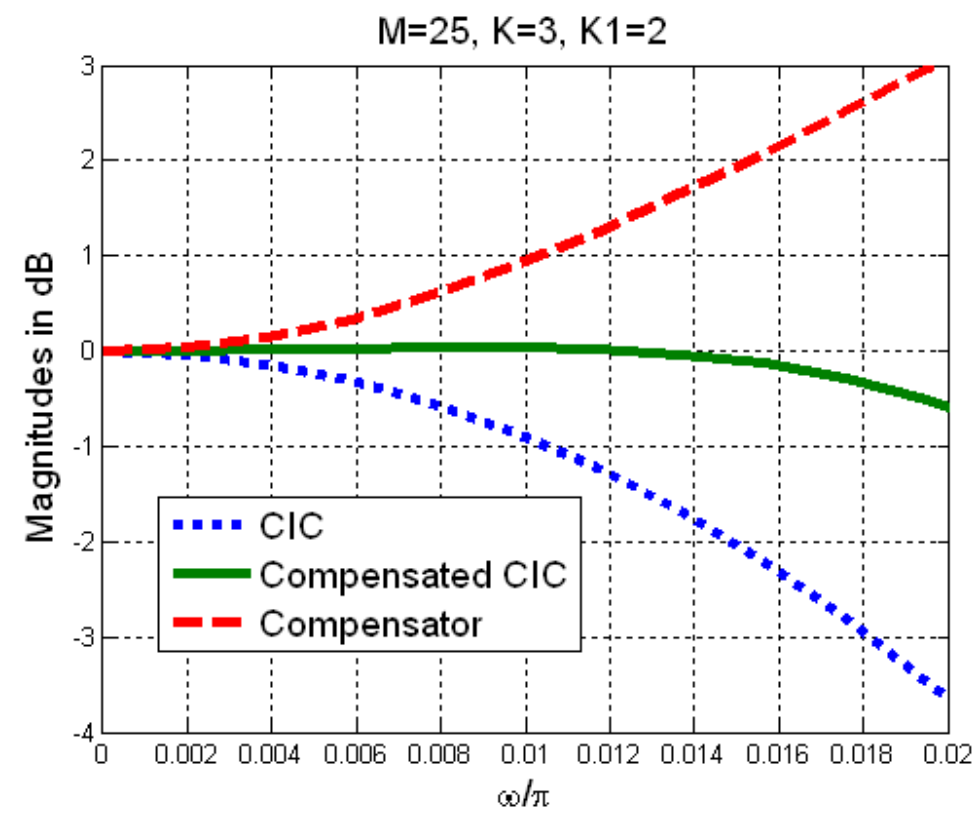

Fig. 5. Wideband CIC compensation using the Compensation method 1. 
The initial value of the parameter $a$ is 1 and the value is adjusted in order to satisfy

$$
\begin{aligned}
& \max \left\{\left|1-H_{c}(\omega) H(\omega)\right|\right\}=d_{p} \leq \delta_{p} \\
& \omega \in\left[0, \omega_{p}\right] .
\end{aligned}
$$

Let us indicate how the coefficients $a$ and $b$ depend on $M$ for a given $K$. To this end, considering that for a small value of $\varphi, \sin (\varphi) \sim \varphi$, and knowing that $M » 1$, we have

$$
\begin{aligned}
& b \approx 0.5\left[1-\frac{\alpha \pi^{K}}{4^{K} \sin ^{K}(\pi / 4)}\right] . \\
& a \approx 1-\left[1-\frac{\alpha \pi^{K}}{4^{K} \sin ^{K}(\pi / 4)}\right] .
\end{aligned}
$$

From (20), (24) and (25) it follows the desirable characteristic, that the compensator does not depend on the decimation factor $M$ but only on the parameter $K$, is satisfied. Next, the coefficients (24) and (25) are rounded to the nearest integer, using the rounding constant $\mathrm{r}=2^{-5}$, resulting in

$$
H_{p}\left(z^{M}\right)=S\left[B z^{-M}+A z^{-2 M}+B z^{-3 M}\right]
$$

where $S$ is the scaling factor and $A$ and $B$ are integers, which can be implemented using only adders and shifts. Consequently the decimator (26) is also multiplierless.

We also note that the compensator can be moved to a lower rate using the multirate identity, (Jovanovic Dolecek, 2002), thereby becoming a second order filter,

$$
H_{p}\left(z^{M}\right)=S\left[B z^{-1}+A z^{-2}+B z^{-3}\right] .
$$

Table 2 shows the values for $S, A$ and $B$ for different values of $K$. The total number of additions and the corresponding passband deviations are also shown.

\begin{tabular}{|c|c|c|c|c|c|}
\hline $\boldsymbol{K}$ & $\boldsymbol{S}$ & $\boldsymbol{B}$ & $\boldsymbol{A}$ & $\boldsymbol{d}_{p}[\mathrm{~dB}]$ & Number of additions \\
\hline 1 & $2^{-4}$ & -1 & $2^{4+2^{1}}$ & 0.142 & 3 \\
\hline 2 & $2^{-3}$ & -1 & $2^{3}+2^{1}$ & 0.234 & 3 \\
\hline 3 & $2^{-4}$ & $-2-2^{0}$ & $2^{4+2^{2}+2^{1}}$ & 0.297 & 5 \\
\hline 4 & $2^{-2}$ & -1 & $2^{2+2^{1}}$ & 0.342 & 3 \\
\hline 5 & $2^{-4}$ & $-2^{2}-2^{0}$ & $2^{4}+2^{3+2^{1}}$ & 0.377 & 5 \\
\hline
\end{tabular}

Table 2. The design parameters.

We make the following observations:

- The maximum number of adders is 5 .

- The passband deviation is less than $\delta_{p}=0.4 \mathrm{~dB}$.

- The smallest deviation is obtained for $K=1,\left(d_{p}=0.142 \mathrm{~dB}\right)$, while the largest is for $K=5$, $\left(d_{p}=0.377 \mathrm{~dB}\right)$.

The method is illustrated in the following examples.

Example 4: We compensate the CIC filter with $M=32$ and $K=4$. The values of $\mathrm{B}, A$, and $S$, from Table 2, are $-1,2^{2+21}$ and $2^{-2}$, respectively. The magnitude responses are illustrated in Fig.6. 
Example 5: We compare the methods (Jovanovic Dolecek, 2009) and (Jovanovic Dolecek \& Dolecek, 2010) for $M=16$ and $K=4$ and 5. The result is shown in Fig. 7. For $K=4$ the methods (Jovanovic Dolecek, 2009) and (Jovanovic Dolecek \& Dolecek, 2010) require 9 and 3 adders , respectively. For $K=5$ the method (Jovanovic Dolecek \& Dolecek, 2010) requires 5 adders whereas the method (Jovanovic Dolecek, 2009) requires 12 adders.

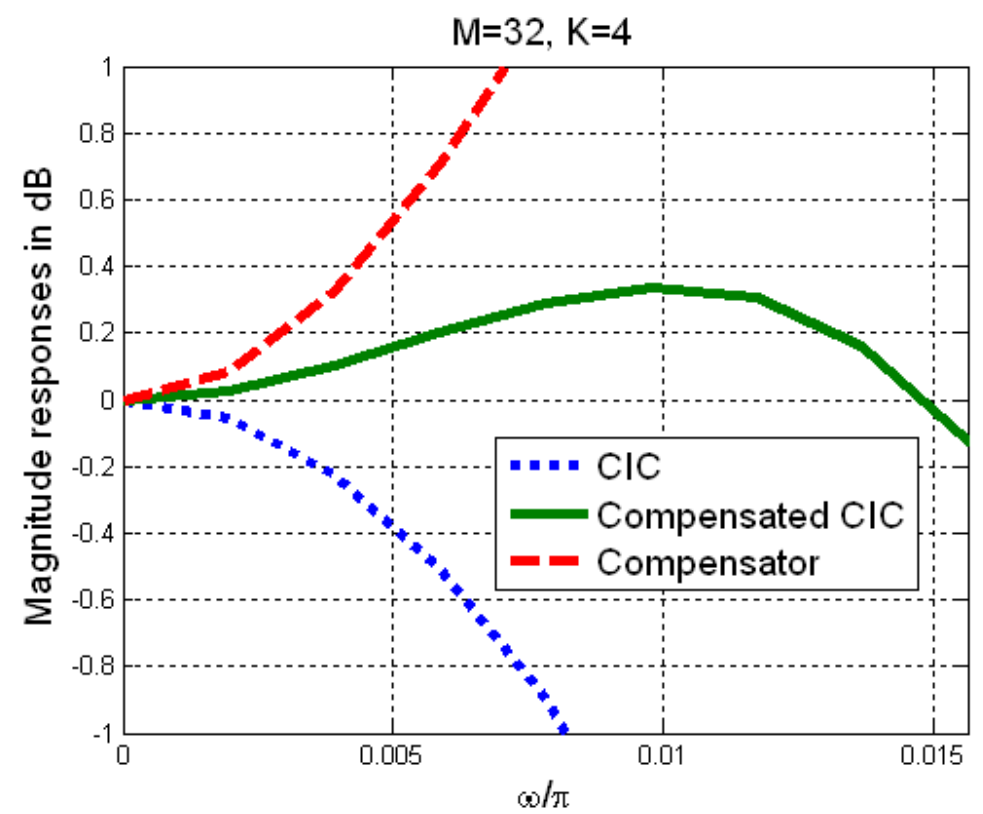

Fig. 6. Wideband CIC compensation using the method (Jovanovic Dolecek \& Dolecek, 2010).

\section{Methods for the stopband improvement}

Presti, (Presti, 2000), introduced the CIC zero rotation and proposed the Rotated Sinc (RS) filter to increase the attenuations and widths in the folding bands. By applying a clockwise rotation of $\beta$ radians to any zero of CIC filter, we obtain the following transfer function

$$
H_{u}(z)=\frac{1}{M} \frac{1-z^{-M} e^{j \beta M}}{1-z^{-1} e^{j \beta}} .
$$

An expression equivalent to (28) is obtained by applying the opposite rotation

$$
H_{d}(z)=\frac{1}{M} \frac{1-z^{-M} e^{-j \beta M}}{1-z^{-1} e^{-j \beta}} .
$$

These two filters have complex coefficients, but they can be cascaded, thus obtaining a filter $H_{r}(z)$ with real coefficients

$$
H_{r}(z)=H_{u}(z) H_{d}(z)=\frac{1}{M^{2}} \frac{1-2 \cos (\beta M) z^{-M}+z^{-2 M}}{1-2 \cos (\beta) z^{-1}+z^{-2}} .
$$




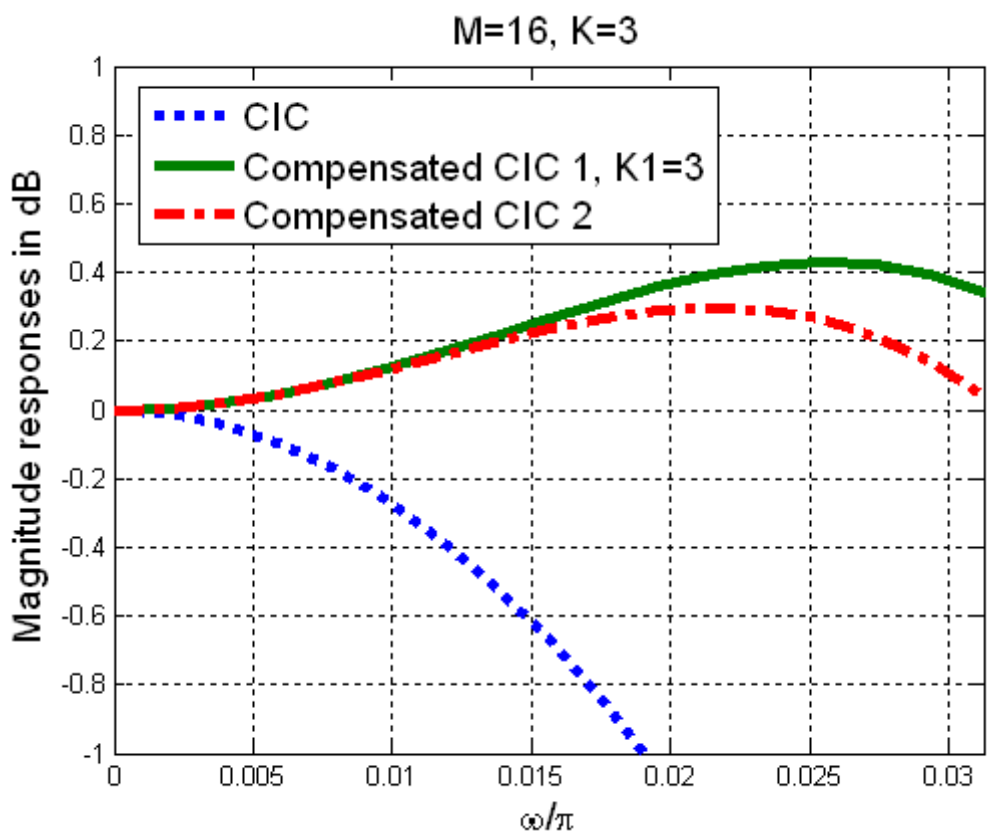

a. $K=3$.

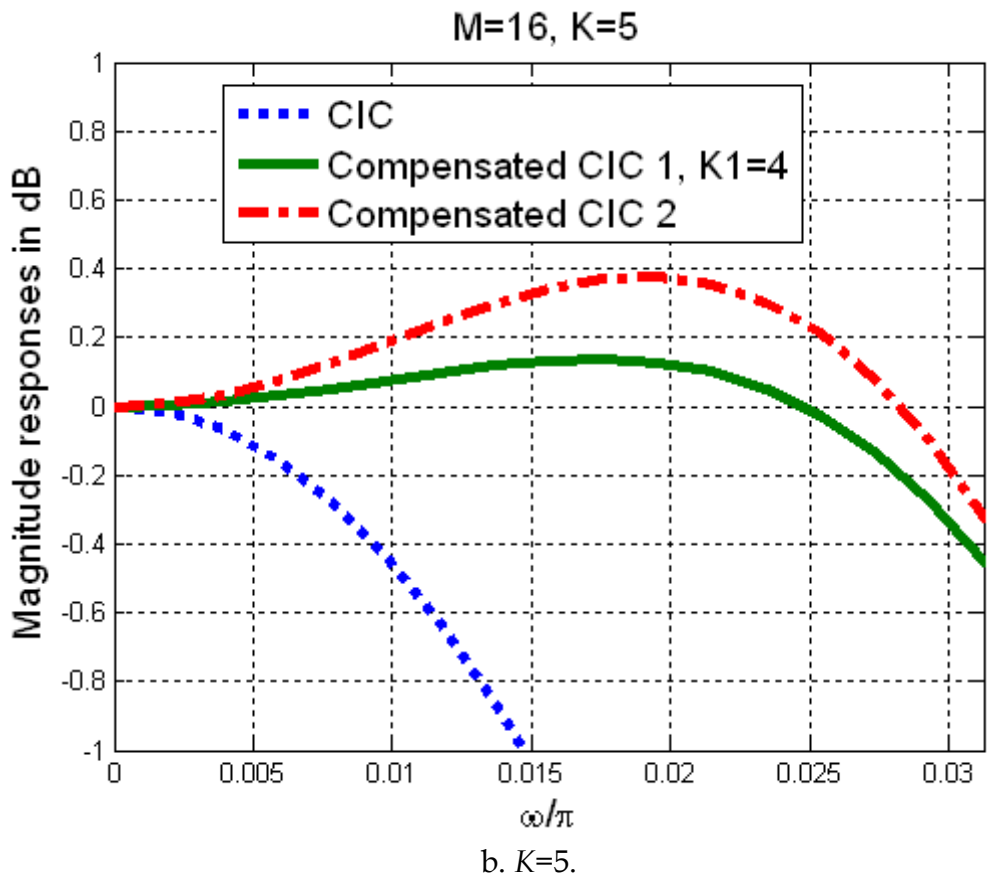

Fig. 7. Comparisons of compensators. 
The cascade of CIC filter and the filter (30) is reffered by Presti as RS filter, $H_{R}(z)$,

$$
H_{R}(z)=H_{\text {comb }}(z) H_{r}(z) .
$$

The magnitude response of this filter is given as

$$
\left|H_{R}\left(e^{j \omega}\right)\right|=\left|\frac{1}{M^{3}} \frac{\sin (\omega M / 2)}{\sin (\omega / 2)}\right|^{K}\left|\frac{\sin ((\omega+\beta) M / 2)}{\sin ((\omega+\beta) / 2)}\right|^{K}\left|\frac{\sin ((\omega-\beta) M / 2)}{\sin ((\omega-\beta) / 2)}\right|^{K} .
$$

Example 6: Using the method Presti, we design the RS filter for $M=16, K=1$, and $\beta=0.0184$. The magnitude response is shown in Fig.8.

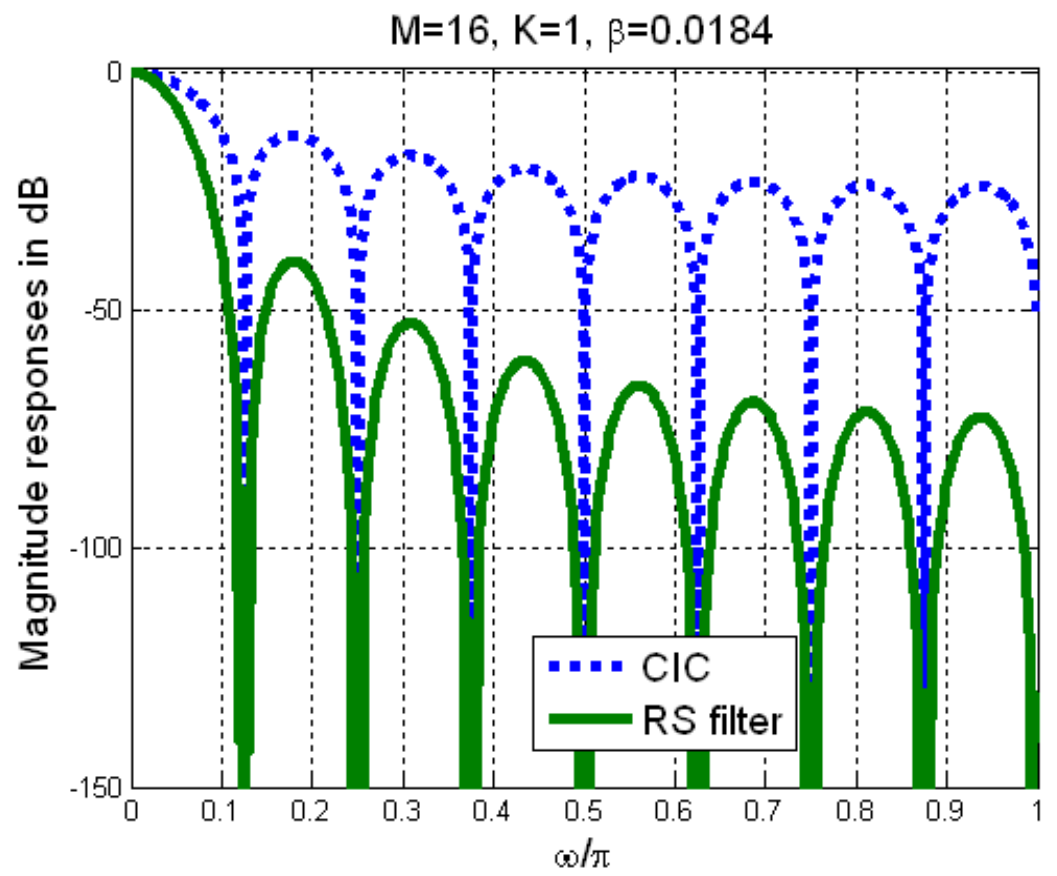

Fig. 8. Illustration of RS filter. (Presti, 2000).

Note that the folding band widths are wider and the attenuations are increased in comparison with the CIC filter. However, the passband droop is increased and additionally RS filter needs two multipliers, one working at high input rate. (See (30)).

In (Jovanovic Dolecek \& Mitra, 2004) the modification of the Presti method has been proposed for the case if $M$ can be represented as a product of two factors

$$
M=M_{1} \mathrm{M}_{2} .
$$

The transfer function (1) can be rewritten as

$$
H(z)=H_{1}^{K_{1}}(z) H_{2}^{K_{2}}\left(z^{M_{1}}\right) .
$$


where

$$
H_{1}(z)=\frac{1}{M_{1}} \frac{1-z^{-M_{1}}}{1-z^{-1}} ; \quad H_{2}\left(z^{M_{1}}\right)=\frac{1}{M_{2}} \frac{1-z^{-M_{1} M_{2}}}{1-z^{-M_{1}}} .
$$

The filter $H_{2}(z)$ can be moved to a low rate which is $M_{2}$ time lesser than the high input rate. Additionally, the polyphase decomposition of the filter $H_{1}(z)$ move all filtering to a lower rate. The corresponding RS filter is modified in such way that it can also be moved to a lower rate.

$$
H_{r m}(z)=H_{u m}(z) H_{d m}(z)=\frac{1}{M_{2}^{2}} \frac{1-2 \cos (\beta M) z^{-M}+z^{-2 M}}{1-2 \cos \left(\beta M_{1}\right) z^{-1}+z^{-2 M_{1}}} .
$$

The modified RS filter is

$$
H_{R m}(z)=H_{c o m b}(z) H_{r m}(z) .
$$

The corresponding magnitude response is

$$
\left|H_{R m}\left(e^{j \omega}\right)\right|=\left|\frac{1}{M} \frac{\sin (\omega M / 2)}{\sin (\omega / 2)}\right|^{K}\left|\frac{\sin ((\omega+\beta) M / 2)}{M \sin \left((\omega+\beta) M_{1} / 2\right)}\right|\left|\frac{\sin ((\omega-\beta) M / 2)}{M \sin \left((\omega-\beta) M_{1} / 2\right)}\right| .
$$

Next example compares the (38) with the RS filter.

Example 7: We use the same design parameters as in Example 6 taking $K_{1}=3$ and $K_{2}=2$ and $M_{1}=M_{2}=4$. The magnitude responses along with the zoom in the first folding band are shown in Fig. 9. Note that the attenuation in the all folding bands except the last one, are improved. Additionally, the filter $H_{r}(z)$ works at a lower rate.

The method in (Jovanovic Dolecek \& Mitra, 2005a) includes the multistage structure and improves deteriorated passband. The generalized approach to the CIC zero-rotation, has been proposed in (Laddomada, 2007), where the generalized comb (GC) has been proposed. An economical class of droop-compensated GC filters has been proposed in (Jovanovic Dolecek \& Laddomada, 2010).

Note the following:

- Folding bands are wider and with increased attenuations comparing with those of the corresponding comb filter.

- $\quad$ The RS filter needs two multipliers, one working at the high input rate.

- During the quantization of the coefficients in RS filter, the pole-zero cancellation can be lost resulting in instability.

- The most critical is the first folding band of a comb filter where the worst case aliasing occurs because it has less attenuation than other folding bands.

To this end in order to solve some of the above mentioned problems we propose to introduce the zero-rotation only in the first folding band yielding in the zero-rotation term (ZRT), (Jovanovic Dolecek, 2010a),

$$
\begin{aligned}
& H_{Z R}(z)=k\left(1-z^{-1} e^{-j \beta}\right)\left(1-z^{-1} e^{j \beta}\right) \\
& =k\left(1-2 \cos (\beta) z^{-1}+z^{-2}\right)
\end{aligned}
$$

where $k$ is the normalizing constant introduced to ensure that the magnitude characteristic is equal to 1 at $\omega=0$. 


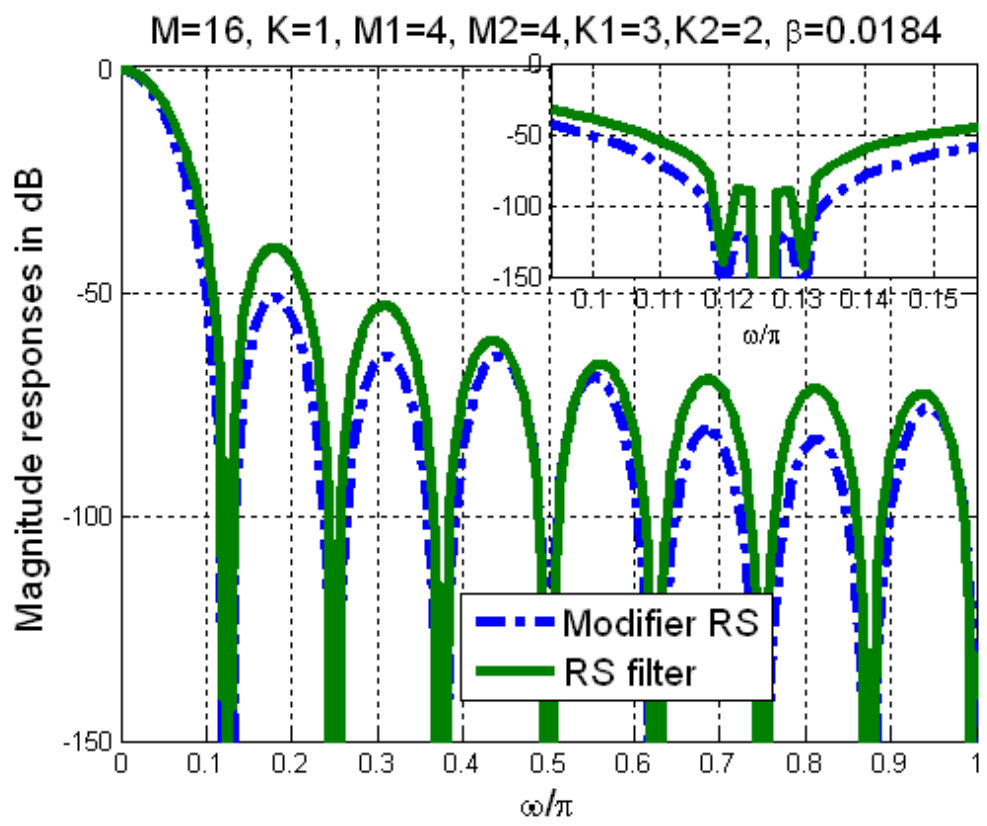

Fig. 9. Comparison of RS and modified RS filters.

Considering that $R$ in (4) is equal to 2 , the pass band is defined by the pass band cutoff frequency

$$
\omega_{p}=\frac{\pi}{2 M} .
$$

The introduced zero must be in the first folding band, near the point where the worst case aliasing occurs, $2 \pi / M-\omega_{p}$,

$$
\beta=\frac{2 \pi}{M}-\frac{\pi}{\left(\beta_{0}+2\right) M},
$$

where $\beta_{0}$ is the term which approaches slightly zero from the left end of the first folding band to the right position, within the first folding band. Typical value for $\beta_{0}=0.99$. The normalized constant $k$ is,

$$
k=\frac{1}{2-2 \cos \left(\frac{2 \pi}{M}-\frac{\pi}{\left(\beta_{0}+2\right) M}\right)} .
$$

Using (41) the cascade of the combs from (1) and the ZRT (39) is given as

$$
H_{C O M B, Z R}=\left[\frac{1}{M} \frac{1-z^{-M}}{1-z^{-1}}\right]^{K} \times k\left(1-2 z^{-1} \cos \left(\frac{2 \pi}{M}-\frac{\pi}{\left(\beta_{0}+2\right) M}\right)+z^{-2}\right) .
$$


The first folding band is wider than the CIC first folding band. However, the side lobes are increased and the pass band droop is also increased. To decrease attenuation in all other folding bands we propose to use cascade of the expanded cosine filters,

$$
H_{\operatorname{COS}}(z)=\prod_{k}\left[\frac{1}{2}\left(1+z^{-N_{k}}\right)\right]^{K_{k}}
$$

resulting in

$$
H_{\mathrm{COMB}, \mathrm{ZR}, \mathrm{COS}}(z)=H_{\mathrm{COMB}}(z) H_{\mathrm{ZR}}(z) H_{\mathrm{COS}}(z) \text {. }
$$

The method is illustrated in the following example.

Example 8: Let us consider CIC filter with $M=12, K=5$ and $K=6$. The expanded cosine filters are

$$
H_{\text {COS }}(z)=\prod_{k=1}^{6}\left[\frac{1}{2}\left(1+z^{-N_{k}}\right)\right]^{K_{k}}, N_{k}=k ; K_{1}=2 ; K_{k}=1, \text { for } k=2, \ldots, 6 ;
$$

The magnitude responses along with the passband zoom are shown in Fig.10. Note that the first folding band is wider and that exhibits higher attenuation than the first folding bands of CIC filters for $K=5$ and 6. See (Jovanovic Dolecek, 2010a) for more details about the choice of design parameters and the multiplierless design.

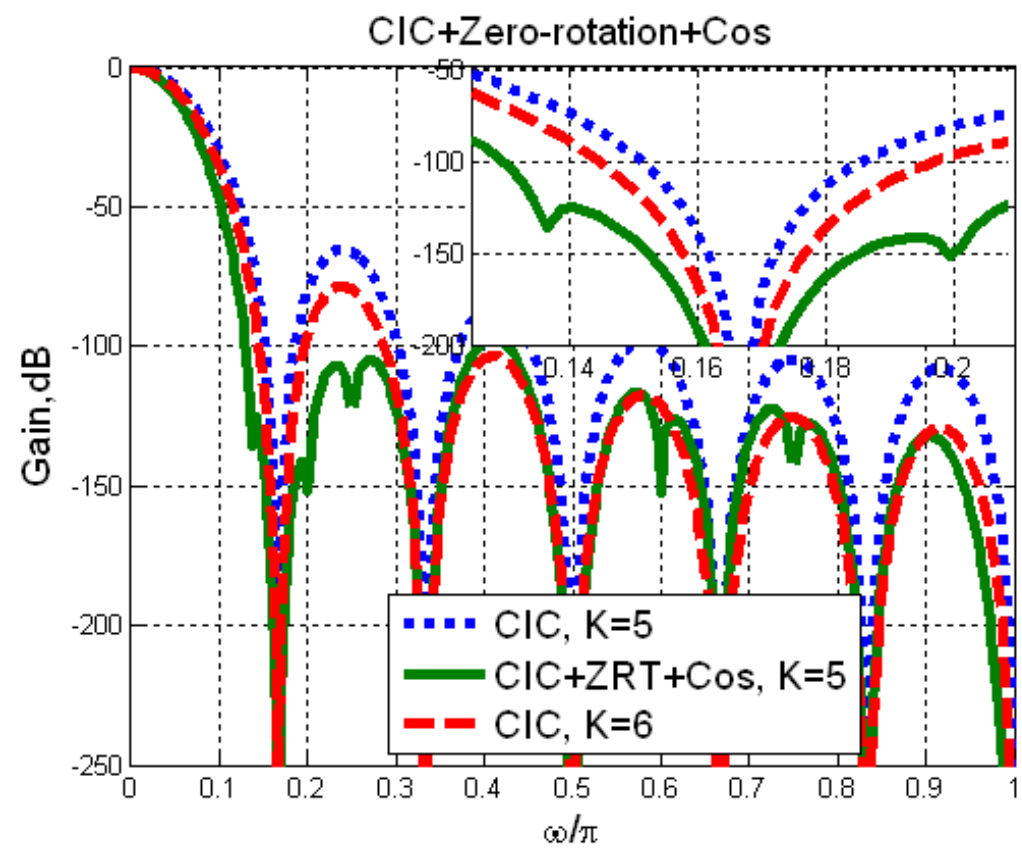

Fig. 10. Illustration of method (Jovanovic Dolecek, 2010a). 
Another approach to improving the CIC stopband characteristic has been proposed in (Jovanovic Dolecek \& Diaz-Carmona, 2005). The method is based on the cosine prefilters introduced in (Lian \& Lim, 1993). Recently, the method based on the extended search of cyclotomic polynomials has been also proposed (Laddomada at al, 2011).

\section{Methods for the passband and stopband improvement}

In this section we consider some methods applied for the simultaneous improvement in the CIC passband and stopband. The pioneer work has been presented in (Kwentus \& Willson, 1997), where the sharpening technique originally introduced by (Kaiser \& Hamming, 1977) was applied. The sharpening technique uses the sharpening polynomials to improve the passband and the stopband characteristics of the symmetrical FIR (Finite impulse response) filter. Kwentus and Willson used the polynomial $H_{s h}=3 H^{2}-2 H^{3}$, where $H$ is the CIC filter (1) and $K=K_{1}$. The corresponding magnitude response of the sharpened CIC filter is

$$
\left|H_{\text {shcomb }}\left(e^{j \omega}\right)\right|=\left|3\left(\frac{1}{M} \frac{\sin (\omega M / 2)}{\sin (\omega / 2)}\right)^{2 K_{1}}-2\left(\frac{1}{M} \frac{\sin (\omega M / 2)}{\sin (\omega / 2)}\right)^{3 K_{1}}\right| .
$$

The method is illustrated in the Example 9.

Example 9: The parameters of the CIC filter are $M=16$ and $K=5$ and $K_{1}=3$. Figure 11a shows the magnitude responses of the sharpened CIC filter and the CIC filter with $K=5$. Figure $11 b$ shows the zooms in the passband and in the first folding band. Note that both the passband and the stopband are improved.

The main drawback of this method is that the sharpening is performed at high input rate. A method where the decimation is split into two stages, and the sharpening is performed only in the second stage considering that the decimation factor $M$ is an even number, has been proposed in (Jovanovic Dolecek \& Mitra, 2003). The method was generalized later for the case where the decimation factor $M$ can be expressed as in (33). The first stage is the less simple CIC filter $\left(M_{1}<M\right)$, which can be implemented either in recursive or non recursive form.

$$
H_{1}(z)=\frac{1}{M_{1}} \frac{1-z^{-M_{1}}}{1-z^{-1}}=\sum_{i=0}^{M_{1}-1} z^{-i} .
$$

In the second stage is the less complex CIC filter, $\left(M_{2}<M\right)$

$$
H_{2}(z)=\frac{1}{M_{2}} \frac{1-z^{-M_{2}}}{1-z^{-1}}
$$

The overall transfer function is

$$
H(z)=H_{1}^{K_{1}}(z) \operatorname{Sh}\left\{H_{2}^{K_{2}}\left(z^{M_{1}}\right)\right\},
$$

where Sh\{.\} means sharpening of $\{$.$\} , and$

$$
K_{1} \geq 2 K_{2} .
$$




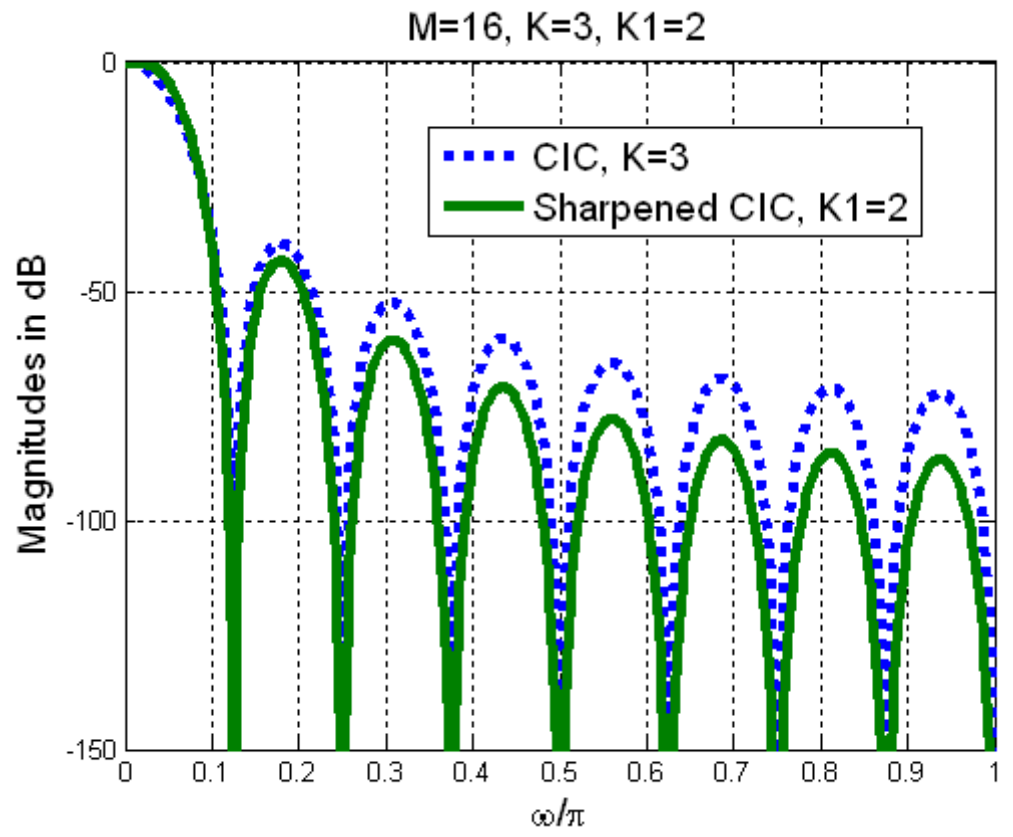

a. Overall magnitude responses.

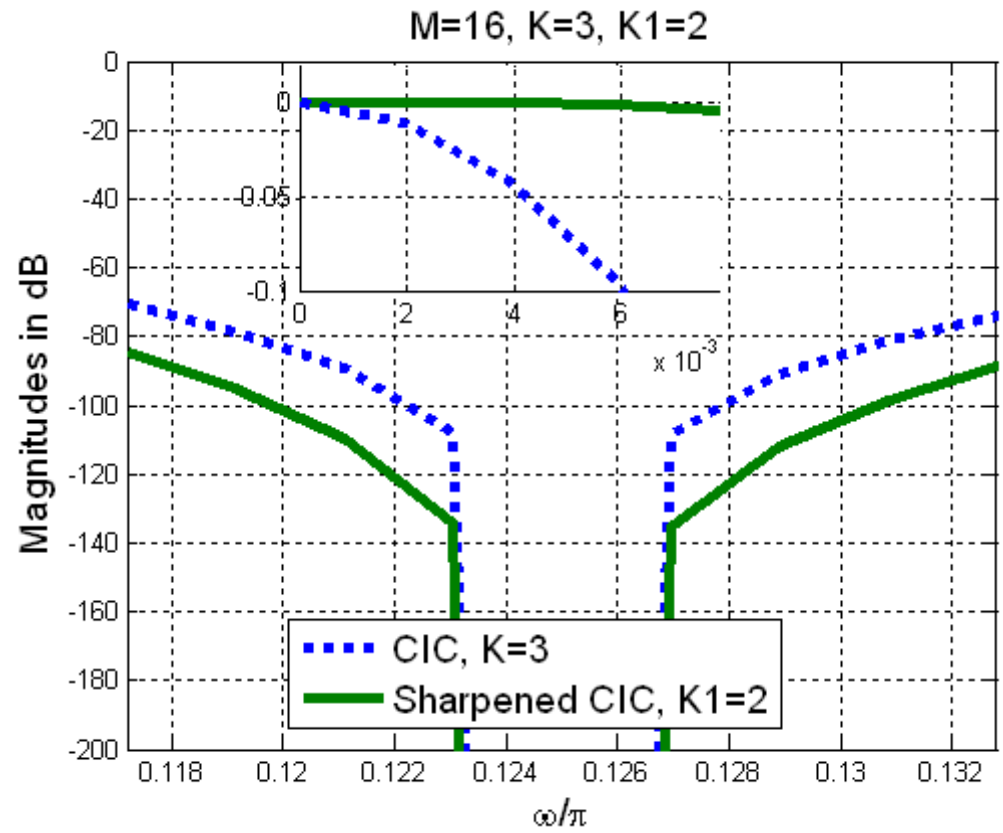

b. Passband and the first folding band zooms.

Fig. 11. Illustration of sharpening method. 
The corresponding magnitude response is

$$
\begin{aligned}
& \left|H\left(e^{j \omega}\right)\right|= \\
& \mid\left(\frac{1}{M_{1}} \frac{\sin \left(\omega M_{1} / 2\right)}{\sin (\omega / 2)}\right)^{K_{1}}\left\{3 \times\left(\frac{1}{M_{2}} \frac{\sin (\omega M / 2)}{\sin \left(\omega M_{1} / 2\right)}\right)^{2 K_{2}}-2 \times\left(\frac{1}{M_{2}} \frac{\sin (\omega M / 2)}{\sin \left(\omega M_{1} / 2\right)}\right)^{3 K_{2}}\right\} .
\end{aligned}
$$

Next examples (10) and (11) illustrate the method.

Example 10: Consider $M=16$ and $M_{1}=M_{2}=4$. The parameters $K_{1}$ and $K_{2}$ are respectively 5 , and 2 , and $K=4$. The magnitude responses and the pasband zoom are shown in Fig.12.

In the following example we compare the original sharpening method with the modified sharpening method (Jovanovic Dolecek \& Mitra, 2005b).

Example 11. We compare the modified sharpening method with the original sharpening method, considering $M=16$ and $K=4$. In the modified sharpening $M_{1}=M_{2}=4, K_{1}=5$ and $K_{2}=4$. Figure 13 shows the magnitude responses and the corresponding passband zoom. Note that the original sharpening has better passband characteristic while the modified sharpening method has higher attenuations in the folding bands.

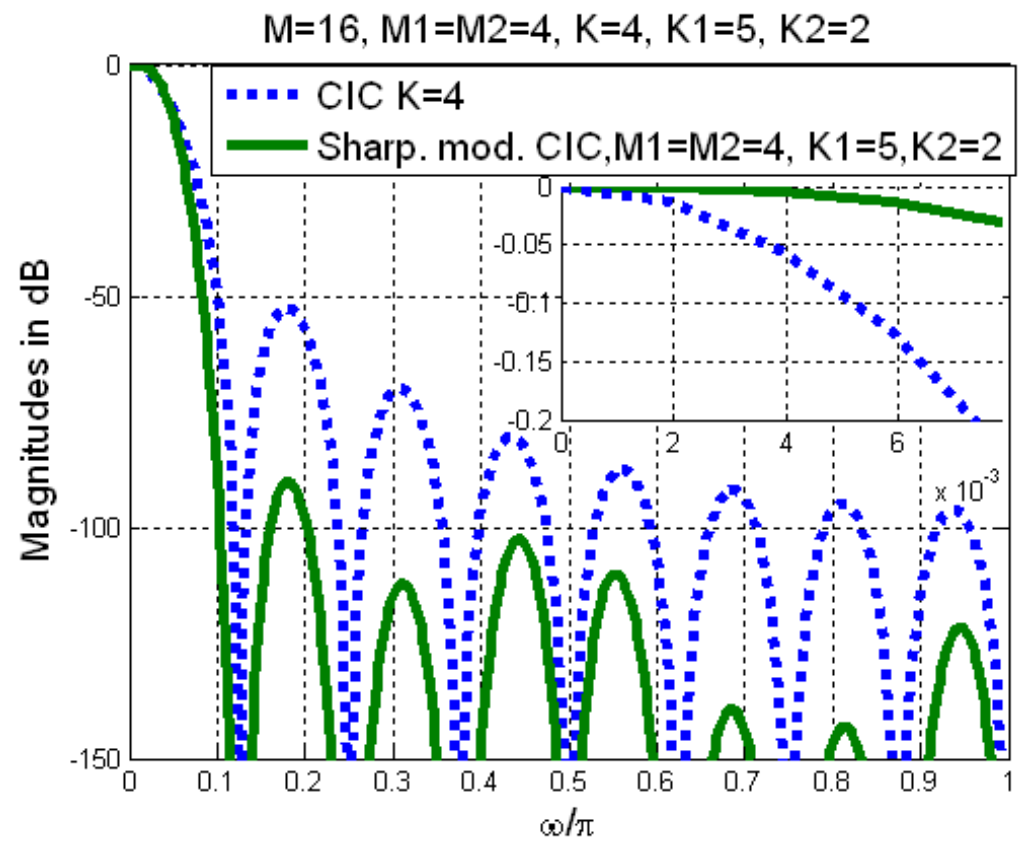

Fig. 12. Modified sharpening and CIC filters magnitude responses. 
The number of authors presented different modifications of sharpening method, like (Jovanovic Dolecek, 2010b), (Laddomada \& Mondin, 2004), (Jovanovic Dolecek \& Harris, 2009). In (Jovanovic Dolecek \& Mitra, 2010), the two-stage CIC filter with the compensator (10) has been proposed.

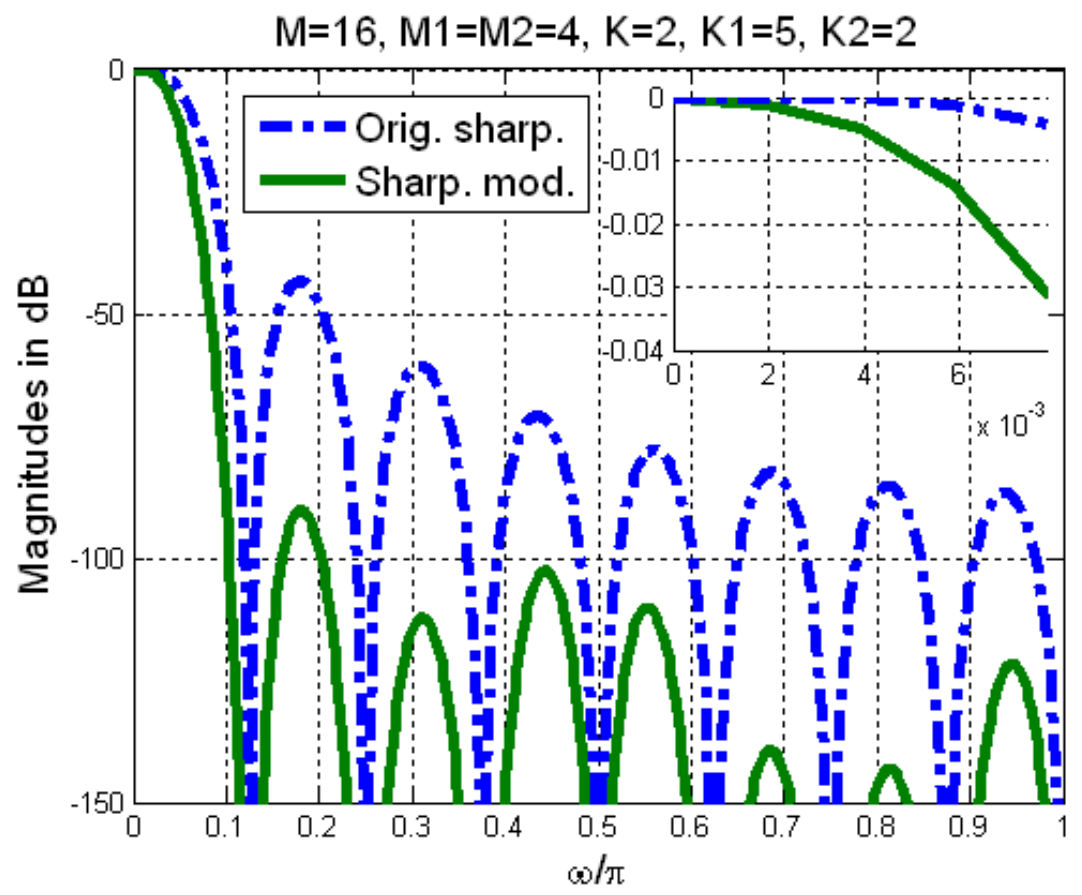

Fig. 13. Comparison of original and modified sharpening method.

The procedure of the design is given in the following steps:

1. For a given $M$ choose the value $M_{1}$, in a such way that the factors $M_{1}$ and $M_{2}$ are close to each other in values, such that $M_{1} \leq M_{2}$ to obtain the filters (48) and (49).

2. Choose the number of the stages $K_{1}$ and $K_{2}$ depending of the desired alias rejection (see Table 3 for tentative values).

3. For given $K_{1}$ and $K_{2}$, choose value of $b$ according to Table 3 .

\begin{tabular}{|c|c|c|}
\hline Parameters $\left(\boldsymbol{K}_{\mathbf{1}}, \boldsymbol{K}_{\mathbf{2}}\right)$ & $\mathbf{A}$ in $\mathbf{d B}$ & $\boldsymbol{b}$ \\
\hline$(2,2)$ & -46.5 & 2 \\
\hline$(2,3)$ & -68.75 & 1 \\
\hline$(3,4)$ & -92.25 & 1 \\
\hline$(4,5)$ & -115 & 0 \\
\hline$(4,6)$ & -139.34 & 0 \\
\hline
\end{tabular}

Table 3. Parameters of design. 
This method is illustrated in the following example.

Example 12: We consider the decimator with $M=16$ and at least $130 \mathrm{~dB}$ worst-case aliasing attenuation. We choose $M_{1}=M_{2}=4$. From Table 3 we get $K_{1}=4, K_{2}=6$ and $b=0$. The method is compared with the two-stage sharpening with $K_{1}=4$ and $K_{2}=2$ in Fig.14. Note that the twostage compensated method has better characteristics.

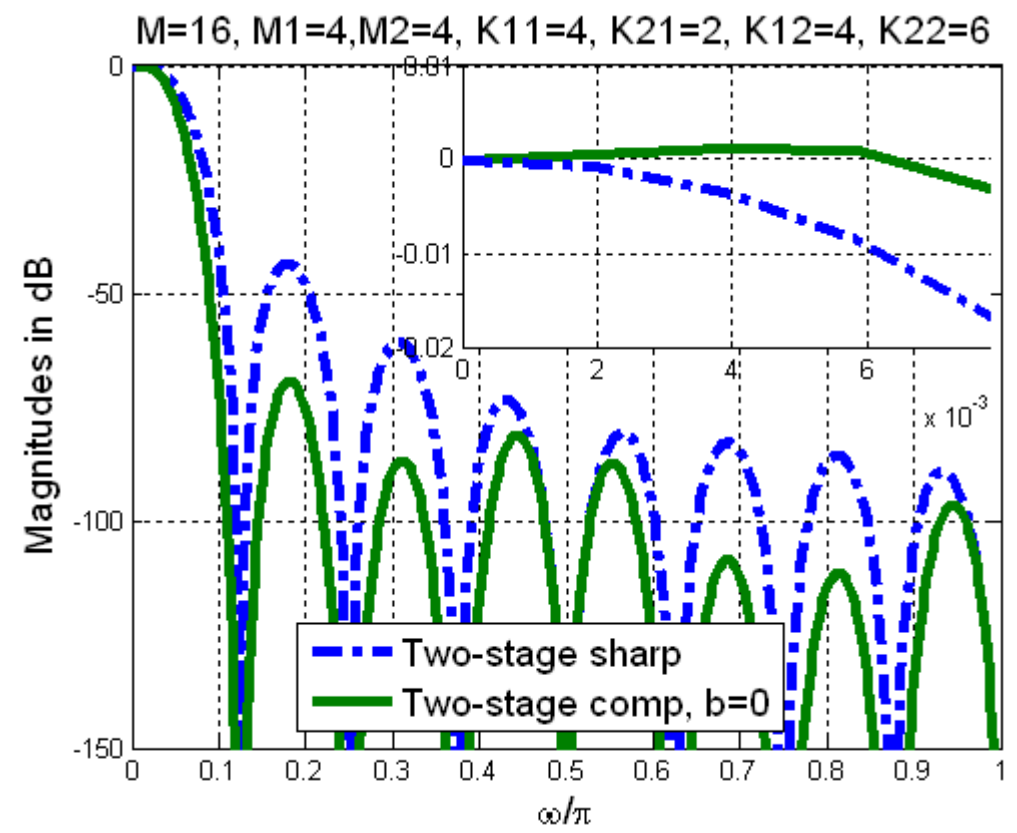

Fig. 14. Comparison of two-stage methods: sharpening and compensated.

\section{Conclusion}

This chapter presents different methods that have been proposed to improve the magnitude characteristics of the CIC decimator. Particularly, the methods are divided into 3 groups depending if the improvement is only in the passband, the stopband or in both i.e. passband and stopband. Only a few methods in each group are described and illustrated in examples. All examples are done in MATLAB and programs can be downloaded from the INAOE web page $w w w-e l e c . i n a o e p . m x /$ paginas_personales/gordana.php.

The CIC filter implementation, which is another important issue concerning the CIC filter, was not considered in this chapter.

\section{Acknowledgment}

Authors thank to CONACYT and to the Institute INAOE for the support. 


\section{References}

Aboushady, H. et al. (2001). Efficient Polyphase Decomposition of Comb Decimation Filters in Sigma Delta Analog-to Digital Converters, IEEE Transactions on Circuits and Systems II, Vol. 48, No. 10, (October 2001), pp. 898-905, ISSN 1057-7130.

Fernandez-Vazquez, A. \& Jovanovic Dolecek, G. (2009). A General Method to design GCF Compensation Filter, IEEE Transactions on Circuits and Systems II: Express Brief, Vol. 56, No. 5, (May 2009), pp. 409-413, ISSN 1549-7747.

Fernandez-Vazquez, A. \& Jovanovic Dolecek, G. (2011). An L2 Design of GCF Compensation Filter, Signal Processing, (Elsevier), Vol. 91, No. 5, (May 2011), pp. 1143-1149, ISSN 0165-1684.

Gao, Y. et al. (2000). A Comparison Design of Comb Decimators for Sigma-Delta Analogto Digital Converters, Analog Integrated Circuits and Signal Processing, Vol. 22, No. 1, (January 2000), pp. 51-60, ISSN 0925-1030.

Hogenauer, E. (1981). An Economical Class of Digital Filters for Decimation and Interpolation, IEEE Transactions Acoustic, Speech and Signal Processing, Vol. ASSP29, (Apr.1981), pp. 155-162, ISSN 0096-3518.

Jovanovic Dolecek, G. (Editor), (2002). Multirate Systems, Design and Applications, Idea Group Publishing, ISBN 1-930708-30-0, Hershey, USA, 2002.

Jovanovic Dolecek, G. \& Mitra, S. K. (2003). Efficient Sharpening of CIC Decimation Filter, Proceedings 2003 International Conference on Acoustics, Speech, and Signal Processing ICASSP 2003, pp.VI.385-VI.388, ISBN 0-7803-7664-1, Hong Kong, China, April, 610, 2003.

Jovanovic Dolecek, G. \& Mitra, S. K. (2004). Efficient Multistage Comb-Modifed Rotated Sinc (RS) decimator. Proceedings XII European Signal Processing Conference EUSIPCO-2004, pp. 1425-1428, ISBN 0-7803-7664-1, Vienna, Austria, September, 6-10, 2004.

Jovanovic Dolecek, G. \& Mitra, S. K. (2005a). A New Multistage Comb-modified Rotated Sinc (RS) Decimator with Sharpened Magnitude Response, IEICE Transactions on Information and Systems: Special Issue on Recent Advances in Circuits and Systems, Vol. E88-D, No. 7, (July 2005),pp. 1331-1339, ISSN 105-0011.

Jovanovic Dolecek, G. \& Mitra, S. K. (2005b). A New Two-Stage Sharpened Comb Decimator', IEEE Transactions on Circuits and Systems-I: Regular papers, Vol. 52, No. 7, (July 2005), pp. 1416-1420, ISSN 1057-7122.

Jovanovic Dolecek, Diaz-Carmona, J. (2005). A New Cascaded Modified CIC-Cosine Decimation, Proceedings 2005 IEEE International Symposium on Circuits and Systems, ISCAS 2005, pp. 3733-3736, ISBN 0-7803-8835-6, Kobe, Japan, May 23-26, 2005.

Jovanovic Dolecek, G. \& Mitra, S. K. (2008), Simple Method for Compensation of CIC Decimation Filter, Electronics Letters, Vol. 44, No. 19, (September 11, 2008), ISSN 0013-5194.

Jovanovic Dolecek, G. (2009). Simple Wideband CIC Compensator, Electronics Letters, Vol. 45, No. 24, (November 2009), pp. 1270-1272, ISSN 0013-5194. 
Jovanovic Dolecek, G. \& Harris, F. (2009). Design of Wideband Compensator Filter for a Digital IF Receiver", Digital Signal Processing, (Elsevier), Vol. 19, No. 5, (Sept, 2009) pp. 827-837, ISSN 1051-2004.

Jovanovic Dolecek, G. (2010a). Simplified Rotated SINC (RS) Filter for Sigma-Delta A/D Conversion, Proceedings of International Conference on Green Circuits and Systems ICGCS 2010, pp. 283-288, ISBN 978-1-4244-6877-5, Shangai, China, June, 21-23, 2010.

Jovanovic Dolecek, G. (2010 b). Low Power Sharpened Comb Decimation Filter, Proceedings of International Conference on Green Circuits and Systems ICGCS 2010, pp.226-229, ISBN 978-1-4244-6877-5, Shangai, China, June , 21-23, 2010.

Jovanovic Dolecek, G. \& Dolecek, L. (2010). Novel Multiplierless Wide-Band CIC Compensator, Proceedings 2010 IEEE International Symposium on Circuits and Systems ISCAS 2010, pp. 283-288, ISBN 978-1-4244-6877-5, Paris, France, May 30th-June 2nd, 2010.

Jovanovic Dolecek, G. \& Laddomada, M. (2010). An Economical Class of DroopCompensated Generalized Comb Filters: Analysis and Design, IEEE Transactions on Circuits and Systems II: Express Brief, Vol. 51, No. 4, (April 2010), pp. 275-279, ISSN 1549-7747.

Jovanovic Dolecek, G. \& Mitra, S. K. (2010). IET Signal Processing, Vol. 4, No. 1, (March (2010), pp. 22-29. ISSN 1751-96-75.

Kaiser, F. \& Hamming, R.W. (1977). Sharpening the Response of a Symmetric Nonrecursive Filter by Multiple Use of the Same Filter, IEEE Transactions Acoustic, Speech and Signal Processing, Vol. 25, No. 5, (October 1977), pp. 415-422, ISSN 0096-3518.

Kim, S., et al., (2006). Design of CIC Roll-off Compensation Filter in a W-CDMA Digital Receiver, Digital Signal Processing, (Elsevier), Vol. 16, No. 6, (November 2006), pp. 846-854, ISSN 1051-2004.

Kwentus A. \&. Willson, Jr. A, (1997). Application of Filter Sharpening to Cascaded Integrator-Comb Decimation Filters, IEEE Transactions on Signal Processing, Vol. 45, No. 2, (February 1997), pp. 457-467, ISSN 1057-7122.

Laddomada, M. \& Mondin, M. (2004). Decimation Schemes for Sigma-Delta A/D Converters Based on Kaiser and Hamming Sharpened Filters, IEE Proceedings of Vision, Image and Signal Processing, Vol. 151, No. 4, (August 2004), pp. 287-296, ISSN 1350-245X.

Laddomada, M. (2007). Generalized comb decimation filters for Sigma-Delta A/D converters: Analysis and design, IEEE Transactions on Circuits and Systems-I: Regular papers, Vol. 54, No. 5, (May 2007), pp. 994-1005, ISSN 1057-7122.

Laddomadda, M. Troncoso, D. \& Jovanovic Dolecek, G. (2011). Design of Multiplierless Decimation Filters Using an Extended Search of Cyclotomic Polynomials, IEEE Transactions on Circuits and Systems II: Express Brief, (In press), ISSN 1549-7747.

Lian, J. \& Lim, J.C. (1993). New Prefilter Structure for Designing FIR Filters, Electronic Letters, Vol. 93, No. 12, (May 1993), pp. 1034-1035, ISSN 0013-5194. 
Presti, L. L. (2000). Efficient modified-sinc filters for sigma-delta A/D converters, IEEE Transactions on Circuits and Systems-II: Analog and Digital Signal Processing, Vol. 47, No. 11, (November 2000), pp. 1204-1213, ISSN 1057-7130.

Yeung, K. S. \& Chan, S. C. (2004). The Design and Multiplier-less Realization of Software Radio Receivers with Reduced System Delay, IEEE Transactions on Circuits and Systems-I: Regular papers, Vol. 51, No. 12, (December 2004), pp. 2444-2459, ISSN 1057-7122. 


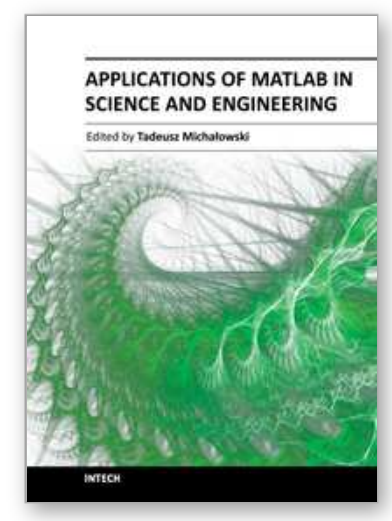

\section{Applications of MATLAB in Science and Engineering \\ Edited by Prof. Tadeusz Michalowski}

ISBN 978-953-307-708-6

Hard cover, 510 pages

Publisher InTech

Published online 09, September, 2011

Published in print edition September, 2011

The book consists of 24 chapters illustrating a wide range of areas where MATLAB tools are applied. These areas include mathematics, physics, chemistry and chemical engineering, mechanical engineering, biological (molecular biology) and medical sciences, communication and control systems, digital signal, image and video processing, system modeling and simulation. Many interesting problems have been included throughout the book, and its contents will be beneficial for students and professionals in wide areas of interest.

\section{How to reference}

In order to correctly reference this scholarly work, feel free to copy and paste the following:

Gordana Jovanovic Dolecek and Javier Diaz-Carmona (2011). On Design of CIC Decimators, Applications of MATLAB in Science and Engineering, Prof. Tadeusz Michalowski (Ed.), ISBN: 978-953-307-708-6, InTech, Available from: http://www.intechopen.com/books/applications-of-matlab-in-science-and-engineering/ondesign-of-cic-decimators

\section{INTECH}

open science | open minds

\author{
InTech Europe \\ University Campus STeP Ri \\ Slavka Krautzeka 83/A \\ 51000 Rijeka, Croatia \\ Phone: +385 (51) 770447 \\ Fax: +385 (51) 686166 \\ www.intechopen.com
}

\author{
InTech China \\ Unit 405, Office Block, Hotel Equatorial Shanghai \\ No.65, Yan An Road (West), Shanghai, 200040, China \\ 中国上海市延安西路65号上海国际贵都大饭店办公楼 405 单元 \\ Phone: +86-21-62489820 \\ Fax: $+86-21-62489821$
}


(C) 2011 The Author(s). Licensee IntechOpen. This chapter is distributed under the terms of the Creative Commons Attribution-NonCommercialShareAlike-3.0 License, which permits use, distribution and reproduction for non-commercial purposes, provided the original is properly cited and derivative works building on this content are distributed under the same license. 